\title{
Evaluating Electron Ionization Mass Spectral Library Search Results
}

\author{
O. David Sparkman \\ O. David Sparkman and Associates, Antioch, California, USA
}

\begin{abstract}
As the size of various collections of electron ionization mass spectra gets larger, there is a continuing and increasing propensity to rely on the results of computerized library searches. The results of these computerized searches do not necessarily account for a spectrum that is produced by the mixture of two or more different compounds. Sometimes the submitted spectrum is not that of a compound whose spectrum is in the library. The quality of the spectrum submitted to the library is often such that the numerical confidence level reported for the search result is so low that it will cause the result to be disregarded. When the sample spectrum is matched against library spectra that have been condensed, the search result can be misleading. Three different examples of mass spectral search results are examined: one, with a high confidence level that the unknown has been identified, but the results are incorrect; one, where the spectrum of the unknown compound is not in the library; one, where consideration of the numerical search results would cause a positive identity not to be confirmed. (J Am Soc Mass Spectrom 1996, 7, 313-318)
\end{abstract}

$\mathrm{E}$ lectron ionization (EI) gas chromatography/mass spectrometry (GC/MS) data of unknown compounds are most always compared against collections of EI mass spectra. Far too often the analysis makes the statement "The library identified this unknown spectrum to be that of ...." Mass spectral libraries and mass spectral library search programs do not identify analytes from mass spectra-people do. The mass spectral library and its associated search program can be a powerful tool in the identity of an unknown. However, like other tools, such as chain saws and firearms, misuse can have devastating results.

A number of factors can affect the quality of a library search result. Among these are the quality of the spectra contained in the database used to search the spectrum of the unknown compound and the search algorithm. These issues have been widely addressed over the last several years [1-3]. Most recently, the efforts to bring about a fully evaluated database of mass spectra have been very well described [3]. By and large, all of the commercially available databases of EI mass spectra contain quality spectra. There are some spectra contained in these databases that are of questionable quality, but this is being resolved by at least one publisher. The quality of the algorithms varies depending on the quality of the spectrum submitted. These issues are best addressed in the study conducted

Address reprint request to O. David Sparkman, 4045 Eagle Ridge Drive, Antioch, CA 94509. by Stein at the National Institute of Standards and Technology [4].

To illustrate the role of the analyst in the use of mass spectral libraries, attempts to identify spectra from three different sets of GC/MS data were explored. The spectra are analyzed by using the NIST/EPA/NIH Mass Spectral Database of 74,827 spectra of 62,235 compounds. The spectra of the unknown compounds were compared against the library by using either the INCOS Library Search Program [5] provided with the Saturn ion trap mass spectrometer (Varian CSB, Walnut Creek, CA) or the NIST Mass Spectral Search Program for Windows, Version 1.1a [6]. The mass spectral database provided with Saturn is of condensed spectra. The maximum number of mass/intensity pairs allowed in any library spectrum is 50. Compounds with fewer than 50 mass/intensity pairs in the database as distributed by NIST are represented by complete spectra, whereas those compounds with more pairs are put through a proprietary condensation routine. An example of this is shown in the comparison of the spectrum of hexachlorobenzene as distributed by NIST and that in the NIST92 Mass Spectral Library distributed by Varian (Figure 1). The condensation algorithm determines the number of peaks in the spectrum that will be included in the library. If there are more than 50 peaks, it will then make a pass through the spectrum to remove peaks at different intervals so that representative peaks remain throughout. If the number of peaks is not reduced to 50 or less, the process is repeated until the desired 
result is achieved. Figure 1 shows the rather dramatic effect this can have. The remaining peaks after several passes of the condensation algorithm are not necessarily the most intense in the original spectrum. Although the condensed spectrum is usually retrieved in a search of the full spectrum of that compound, it may not represent the first "hit" or have a high-enough numerical value to be considered a "good" match.

This use of reduced or condensed spectra is not unique to the implementation of the INCOS algorithm. This was first described by Hertz et al. [7], and the current implementation of McLafferty's probability based matching (PBM) algorithm by Hewlett-Packard (HP) uses condensed spectra [8]. Although the search is carried out against a library of condensed spectra by using the HP PBM algorithm, optionally, the displayed spectra are complete from all libraries distributed by HP except for the Pfleger, Maurer, and Weber library of toxicological compounds, which is only distributed in condensed form. Retained mass/intensity pairs in both of these two cases of condensation, like that of the INCOS algorithm, were based on the significance of the peaks with respect to the mass spectra. These condensations were originally carried out to reduce the amount of storage space required for the mass spectral database. However, that is no longer significant with the large storage capacity of today's desktop computers. The NIST Mass Spectral Search Program uses a presearch of condensed spectra, but the final search is carried out on the entire spectrum.

Examination of the reconstructed chromatographic peak will not always be an indicator that more than one compound is present. The reliability of the numerical confidence factors placed on the search results by the computer, no matter how high, should be questioned with respect to an actual visual evaluation of the mass spectrum.

A good example of how a "good search result" can lead to an incorrect conclusion is shown in the following example of the analysis of a mixture of volatile organic compounds by GC/MS. Although the library search results were well within the positive match

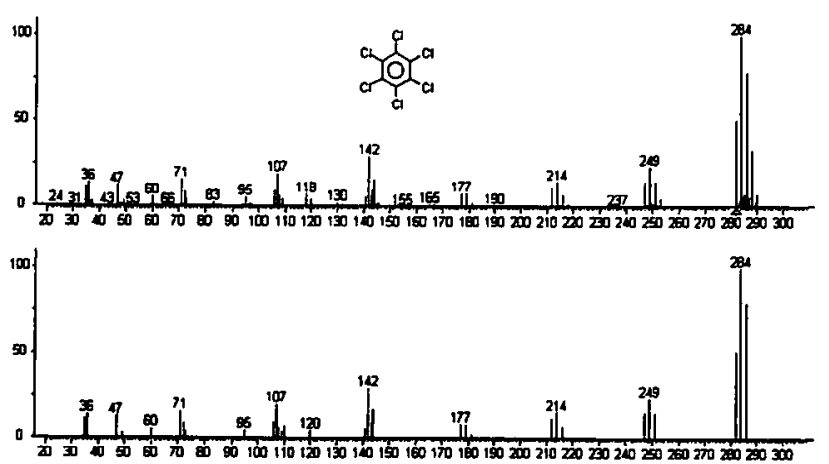

Figure 1. Top: Spectrum as distributed by NIST with 150 peaks. Bottom: Spectrum after condensation as it appears in the NIST 92 library used by the Varian INCOS search program with 31 peaks. criteria set by the description of the program, the identification was incorrect.

\section{Example 1}

A GC/MS analysis was performed on a mixture of volatile organic compounds by using the purge-andtrap method described in US EPA Method 524.2 for the analysis of drinking water. Figure 2 shows a portion of the reconstructed total ion current chromatogram (RTICC) that indicates the presence of at least eight different compounds. It is clear from an examination of the shape of peak 1 that it represents at least two different compounds. There is nothing about the shape of any of the other remaining six peaks that indicates the presence of more than one compound per peak. An identification was attempted of the spectrum that represented the apex of peak 3 in the RTICC (Figure 3). This spectrum was a good candidate for analysis by library search because of the apparent lack of a molecular ion peak.

The spectrum was compared against the NIST/ EPA/NIH Mass Spectral Library by using the INCOS search algorithm in the Saturn software. This algorithm incorporates a presearch of the comparison of the 16 most intense peaks in the unknown spectrum against the 8 most intense peaks in the mass spectral library. The three factors used to judge the reliability of the search results are purity $(P)$, fit $(F)$, and reverse fit (R). Purity is a direct comparison of all the peaks and their intensities in the unknown spectrum and the library spectrum. The fit is a comparison of all the peaks and their intensities in the library spectrum with just those peaks in the unknown spectrum. Peaks in the unknown spectrum that are not in the library spectrum are disregarded as impurities in the calculation of the fit value. The reverse fit (rfit) is a comparison of all the peaks and their intensities in the unknown spectrum with just those peaks in the library spectrum. Peaks in the library spectrum that are not in the unknown spectrum are disregarded [5].

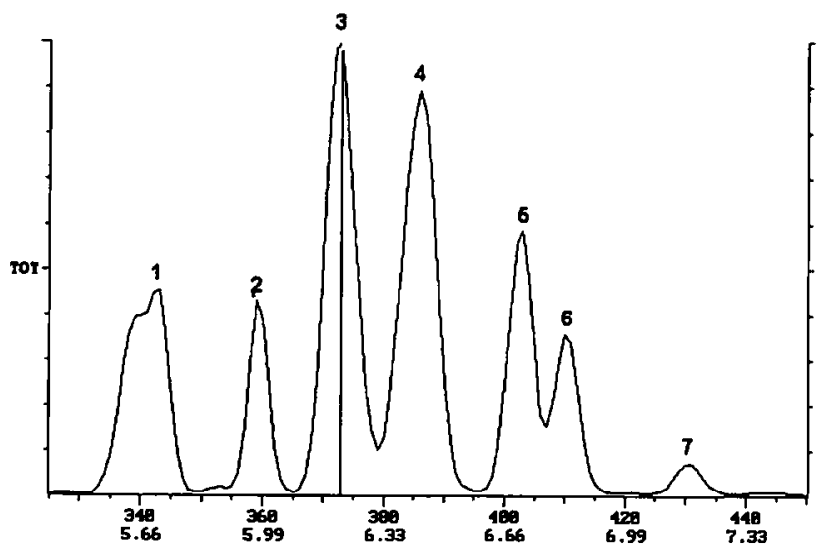

Figure 2. Selected region of a reconstructed total ion current chromatogram from a mixture of volatile organic compounds. 


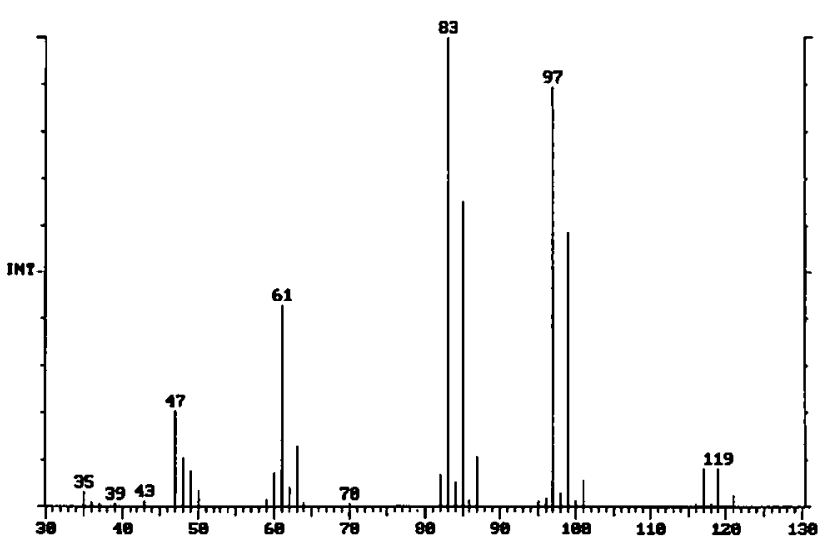

Figure 3. Mass spectrum of scan at apex of peak 3. There appears to be no molecular ion peak in the spectrum.

Figure 4 shows the unknown spectrum at the top followed by the spectra of the first three matches in decreasing order of purity. The first three matches from a comparison with 74,827 spectra are all of the same compound as indicated by the Chemical $\mathrm{Ab}$ stracts Service (CAS) registry number in the upper right corner of each spectrum for the first three "hits."

The confidence levels for these particular library search results are very high. The manual accompanying the software states that values for purity, fit, and rfit of $800-900$ indicate a high probability that the unknown spectrum and the library spectrum are of the same compound [9]. These three values (shown in the upper left of the "best match" spectrum), along with the first three matches all being the same compound, indicate a high probability that the unknown spectrum is that of 1,1,2-trichloroethane. However, a closer examination of the unknown and the "best match" spectrum indicates something much different. Besides differences in the intensity of major peak clusters with nominal mass-to-charge ratio values of 83 and 97, the unknown spectrum has a cluster of peaks with a nominal mass-to-charge ratio value of 117. This cluster does not appear in any of the spectra of the first three matches. The appearance of this cluster indicates that

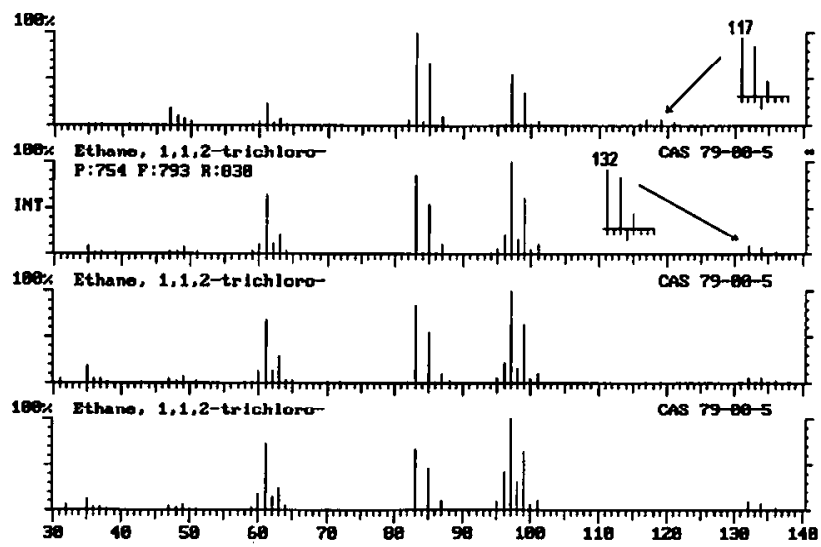

Figure 4. Result of search against the NIST / EPA/NIH library of 74,827 spectra. First three hits are the same compound. it represents an ion that contains three atoms of chlorine. Although all of the spectra of the first three "hits" do not have a cluster of peaks at this nominal mass value, they do have a cluster of peaks with an identical pattern and intensity to that of the unknown's cluster at $m / z 117$ at a nominal mass-to-charge ratio value of 132 (the molecular weight of 1,1,2-trichloroethane). It is obvious that the three best matches are of the same compound, but it is not so obvious that the unknown spectrum is of that same compound.

The RTICC peak was reexamined by displaying scans near the base on each side of the peak. As seen in Figure 5, peak 3 appears to consist of at least two different compounds. To confirm that more than one compound was present, mass chromatograms for the base peak of the two different spectra in Figure 5 are compared in Figure 6. The different retention time (scan number) for the apex of the two mass chromatographic peaks confirms that there are at least two different compounds present.

The scan representing the apex of the $m / z 97$ mass chromatographic peak was subtracted from the scan that represents the apex of the $\mathrm{m} / \mathrm{z} 83$ mass chromatographic peak. This resulted in the spectrum and library search results shown in Figure 7. The reverse was then done and produced the results shown in Figure 8.

Both the examination of the unknown spectrum as compared to the library search spectra and the numeric confidence levels provide the closest to an unambiguous identification of the two compounds in this mixture that can be obtained without doing a direct comparison of the two unknown spectra with spectra obtained on putative samples of the identified compounds.

\section{Example 2}

GC/MS data that have two chromatographic peaks of about the same size resolved by several minutes were examined. The mass spectra that represent the apex of these two chromatographic peaks were identical. These mass spectra contained three major sets of peaks: all

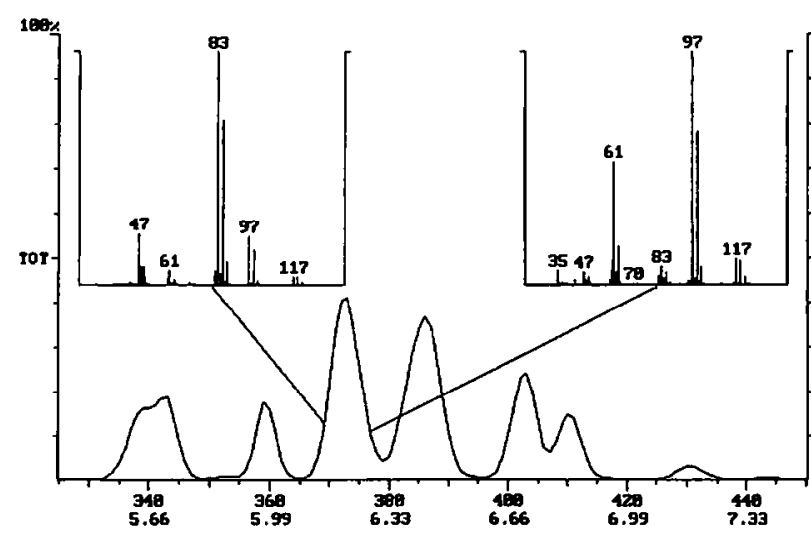

Figure 5. The two different spectra indicate the presence of at least two different compounds. 




Figure 6. Mass chromatograms for the base peak of each of the two spectra confirm the presence of at least two compounds.

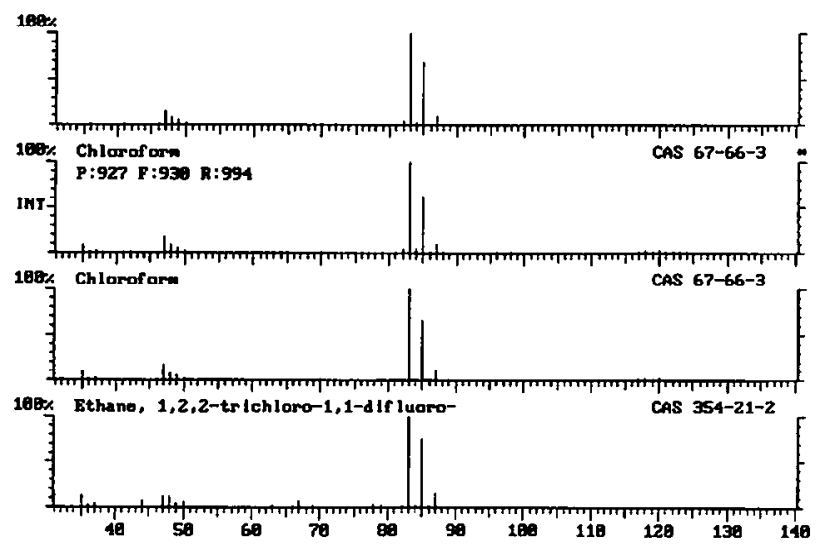

Figure 7. The top spectrum results from subtracting all the peaks in the spectrum with $m / z 97$ as the base peak from the spectrum with $m / z 83$ as the base peak.

had an even nominal mass and each appeared to represent an ion that contained a single atom of chlorine based on isotopic ratios of the nominal mass peak and the $X+2$ peak (top of Figure 9). A search of the spectrum was carried out by using the NIST Mass Spectral Search Program for Windows over the range of $m / z 40-200$. The first spectrum in the "hit list" was interesting. It also had three sets of peaks: each had even nominal mass-to-charge ratio value and each represented an ion that contained a single atom of chlorine (bottom of Figure 9).

The two spectra in Figure 9 have the same general appearance, but there is a significant difference in the

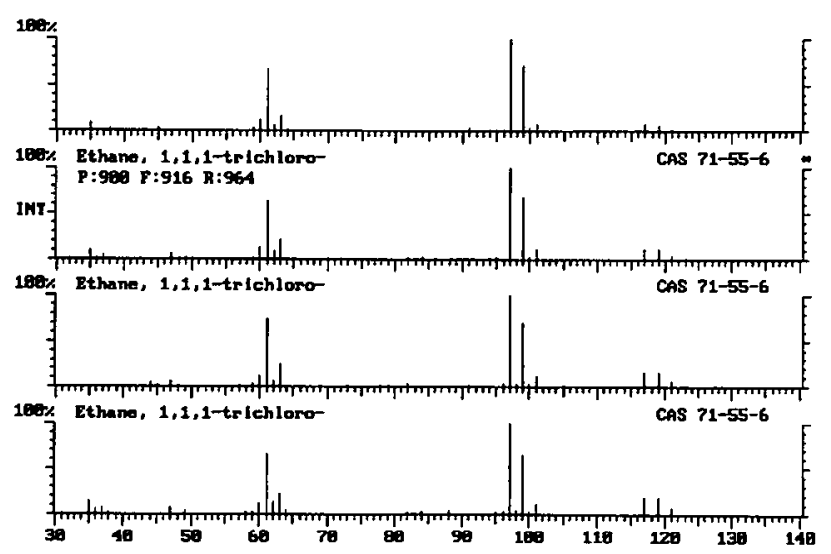

Figure 8. The top spectrum results from subtracting all the peaks in the spectrum with $m / z 83$ as the base peak from the spectrum with $m / z 97$ as the base peak.
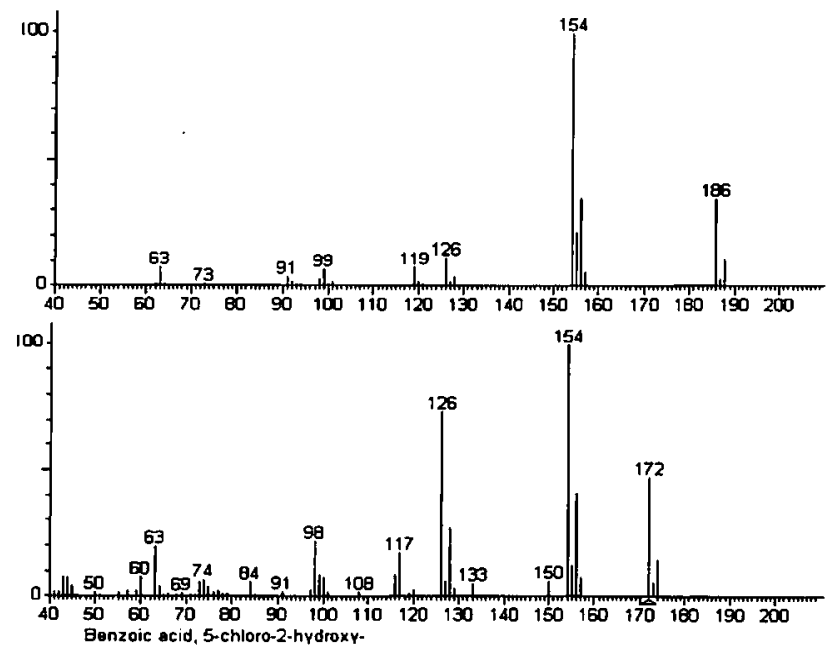

Figure 9. Top: Spectrum of unknown. Bottom: Spectrum of best library match.

peaks that represent the highest mass ions. The nominal mass for the peak that represents the highest mass ion in the spectrum of the unknown is at $m / z 186$ and that of the best library match is at $m / z \quad 172$. This difference of 14 could be due to the addition of a methylene group or it could be due to the difference in the mass of a methoxy as opposed to a hydroxyl group as in the case of a methyl ester. The best match from the library search is 5-chloro-2-hydroxy benzoic acid (5-chlorosalicylic acid). The important factor in the comparison of the two spectra is that both the library spectrum and that of the unknown were dominated by three peaks that appeared to be odd-electron ions, and each ion appeared to contain a single atom of chlorine.

To confirm the identity of the unknown as the methyl ester of chlorosalicylic acid, the spectra of methyl salicylate and salicylic acid were compared (Figure 10). Both spectra showed a dominance of three separate peaks that represent odd-electron ions. Intense peaks represented the molecular ions $(\mathrm{m} / \mathrm{z} 152$
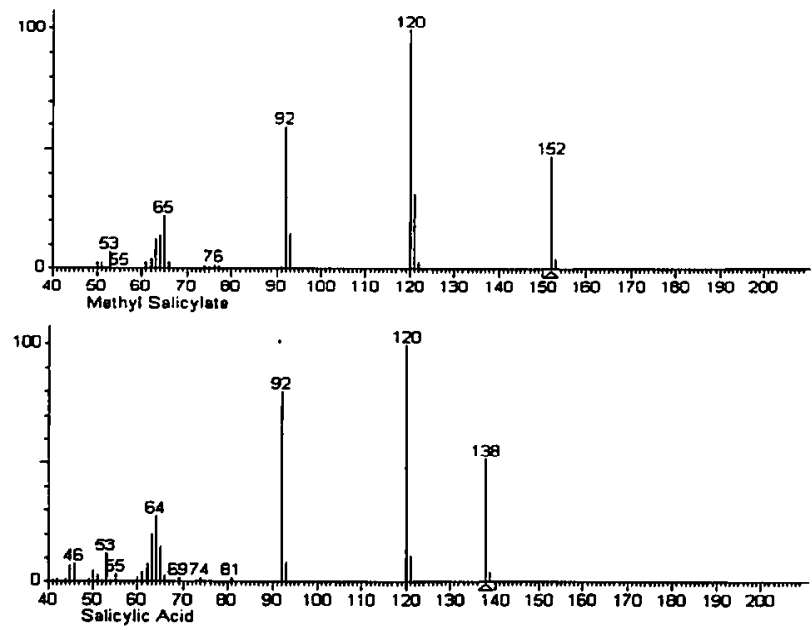

Figure 10. Top: Methyl salicylate. Bottom: Salicylic acid. 
and 138, respectively). Peaks also were present that represent ions that result from the loss of methanol or water, respectively. These ions, which result from the expulsion of a valence-satisfied species from the molecular ion, could then lose $\mathrm{CO}$, which would account for the peaks at $m / z 92$ in each spectrum. These same losses are observed in the spectrum of the corresponding salicylic acid and methyl salicylate. Because the mass-to-charge ratio difference in the two sets of spectra is 35 and the $X+2$ isotopic pattern indicates the presence of a single atom of chlorine, there is a very good probability that the unknown is chloromethyl salicylic acid.

The presence of the chlorine atom at different positions on the ring could result in well-separated chromatographic peaks with the same mass spectrum. Comparison of the spectra of the three possible isomers of chloro phenol indicates that the two unknown chromatographic peaks represent 5-chloromethyl salicylate and 4-chloromethyl salicylate. If the chlorine atom were on the number three carbon atom of the ring, the molecular ion would have a tendency to lose $\mathrm{HCl}$, but peaks that represent the loss of $\mathrm{HCl}$ from the molecular ion are not observed in either of the unknown mass spectra. Notice the intensity of the $X+1$ peak for $m / z 120$ in the methyl salicylate spectrum as compared to that in the spectrum of salicylic acid. This high intensity $X+1$ peak (and corresponding ${ }^{37} \mathrm{Cl}$ isotope peak at $X+3$ ) is observed in the spectra of the two unknown chromatographic peaks. The intensities of these peaks cannot be explained as ${ }^{13} \mathrm{C}$ isotope peaks; they are probably the result of the loss of a $\mathrm{CH}_{3}$ group from the methyl ester part of the molecular ion. This is additional evidence to confirm the identity conclusion.

This use of library search results to identify substructures such as the aromatic ring, the presence of a single atom of chlorine, and the presence of a methyl ester and a hydroxyl group are the bases for computerized substructure identification described by Stein [10].

\section{Example 3}

It is also possible to discard a correct match from a search result due to the numeric quality associated with that result being too low. A good example of this is shown in Figure 11. A component that produced a two-scan-wide gas chromatography peak needed to be identified. This component was present in a semivolatile standard used in US EPA Method 625. The identified compound (Figure 12) has a mass spectrum with very few significant peaks. The low ion current at the time the mass spectrum was taken accounted for the relatively poor quality of the searched spectrum.

With low values for the numeric confirmation of the identity of the analyte and an ambiguous name for the compound of the matched spectrum, the confirmation of the identity was questionable until an examination
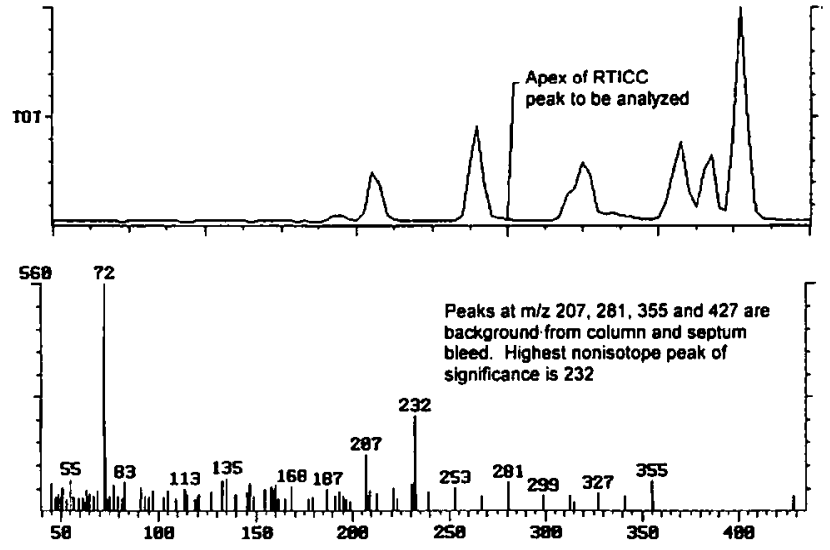

Figure 11. Spectrum which requires identification and its location in the RTICC.

was made of the identified compound's synonyms in the NIST/EPA/NIH Mass Spectral Library (Figure 13).

A herbicide would be expected to be found in a mixture such as that which comprised the sample. With this added piece of information, it is possible to have a higher degree of confidence that the analyte has been identified even with low purity, fit, and rfit values.

\section{Conclusion}

A computerized library search will generally yield an answer. Do not be too quick to accept the answer as an unambiguous identification of the analyte or reject it as not being possible due to low numeric indicators of correctness.

The quality of the results from a computerized library search is dependent on the following criteria:

The quality of the spectra in the library [1-3].

Whether the spectra in the library are searched as complete or condensed spectra.

The search algorithms [4].
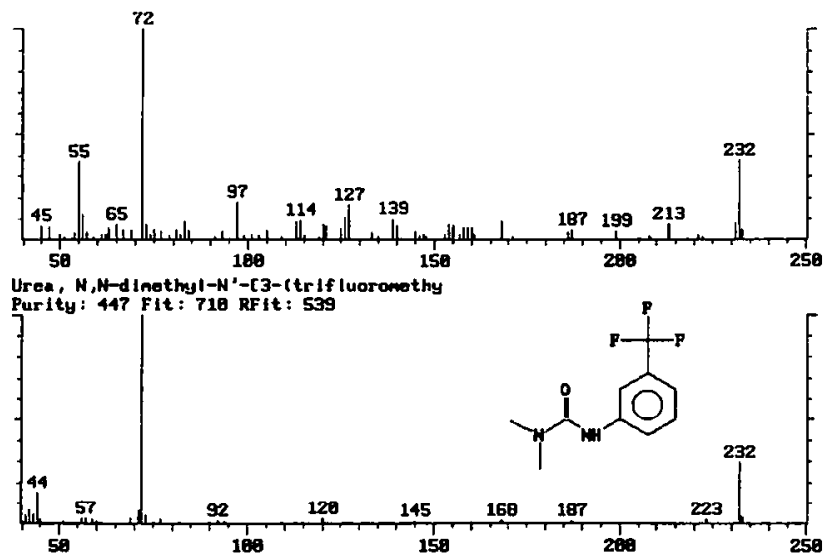

Figure 12. Low values for purity, fit, and reverse fit cause identity to be questioned. 
Urea $\mathrm{N}, \mathrm{N}$-dimethy- $\mathrm{N}-\mathrm{P}$-(trifluoromethy)phenylFormula: C10H11F3N2O

MW: 232 CASt: 2164-17-2 NIST: 52401 10*: 15001 DB: malnlib

Other DBS: Fine. TSCA RTECS, EPA. HODOC, EINECS

Contributor: MASS SPECTROMETRY CENTER. UNIV. OF UTAH ALDRICH T02750-2

Synonyms:

1. Urea 1.1-dimethyl-3.alpha...alpha...alpha-trifluoro-m-tolyn-

2. 62059

3. Clba 2059

4. Cotoran

5. Fluometuron

6. Kotoran

7. Lanex

B. n-(m-Trtfluaramethylphenyl)-N.N'-dimethylurea

9. n-[3-Trtfluoromethylpheny)-N'.N'-dimethylurea

10. Pakhtaran

11. 3-(m-Trilluoromethyiphemy-1.1-dimeth:lurea

12. J-3-Tritluoromethypheny!)-1.T-dimethyurea

13. 1,1-Dimethyt-3-(3-trifluoromethy/phenyl)urea

14. Cottonex

15. Cotoran mult 5owp

16. 1.1-Dimethyt-3-.alphC..alpha. alpha-trtfluoro-m-tolyl) urea

17. Herbicide C-2059

18. Higalcoton

Figure 13. List of synonyms for best match from library search

The quality of the submitted spectrum.

Whether the spectrum of the unknown compound is in the library.

Weakness in any of these areas must be compensated for by careful examination of both the submitted and library spectra. Additional information about the sample's origin and characteristics of the identified compound can be used to increase the confidence of, or even confirm, any identification based on mass spec- tral data alone. People-not mass spectral library search programs-identify compounds based on mass spectral data. The library search program is only a tool used to help in these identifications.

\section{References}

1. McLafferty, F. W.; Stauffer, D. B.; Loh, S. Y. I. Am. Soc. Mass Spectrom. 1991, 2, 438-440.

2. Stein, S. E.; Ausloos, P.; Lias, S. J. Am. Soc. Mass Spectrom. 1991, 2, 441-443.

3. Zaikin, V.; Ausloos, P.; Clifton, C.; Lias, S.; Mikaya, A.; Sparkman, D.; Stein, S. Proceedings of the 43rd ASMS Conference on Mass Spectrometry and Allied Topics; Atlanta, Georgia, May 21-26, 1995; p 140.

4. Stein, S. E. J. Am. Soc. Mass Spectrom. 1994, 5, 316-323.

5. Sokolow, S.; Karnofsky, J.; Gustafson, P. The Finnigan Library Search Program Finnigan Application Report No. 2, March 1978.

6. NIST Mass Spectral Search Program and The NIST/EPA/NIH Mass Spectral Library for Use with Microsoft ${ }^{\circledR}$ Windows $^{\mathrm{TM}}$, Version 1.1a; NIST: Gaithersburg, MD, 1994.

7. Hertz, H. S.; Hites, R. A.; Biemann, K. Anal. Chem., 1971, 43, 681-691.

8. MS ChemStation Handbook, HP 59970C, Rev. 3.2, Publication 59970-90034; Hewlett-Packard Corp.: Palo Alto, CA, June 1988; $p$ 7-4.

9. Saturn GC/MS Reference Manual, 03-914354-40:3; Varian Associates, Inc., 1995; pp 13-19.

10. Stein, S. E. J. Am. Soc. Mass Spectrom. 1995, 6, 644-655. 